\title{
Detection of anti-Myalin and anti-axonal auto-antibodies in Iraqi
} patients with multiple sclerosis

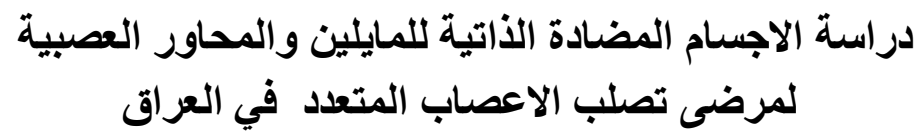

Rasha Majid Abdulamir Al-hemiary,

College of Science for Women/ Baghdad University

رشا ماجد عبد الامير الحميري

كلية العلوم للبنات / جامعة بغداد الامير لمداد

\section{Abstract}

Multiple Sclerosis (MS) is characterized by demyelination of different parts of the central nervous system (CNS) leaving scars (sclerosis) which leaves the neuron and the axis highly attenuated. It is one of the most important inflammatory diseases of the CNS that causes deficits with the progression of neurological disease. This study included 65 MS patients (37 relapsing-remitting (RR), 21 secondary-progressive (SP), 7 primary-progressive (PP) and 50 apparently healthy individuals controls. The results showed low level of autoantibodies to myelin associated glycoprotein (anti-MAG) in MS patients, while anti-axon antibodies had high level. This means that the production of these auto-antibodies in different stages of the disease increase its effect in the diagnosis and the possibility that it had some role in the progress and development of MS, so the use of anti axonal antibodies test in the diagnosis is an important marker.

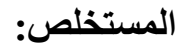

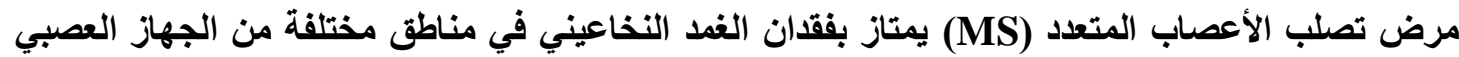

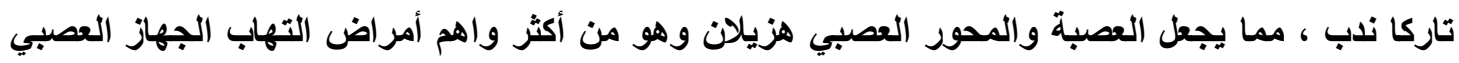

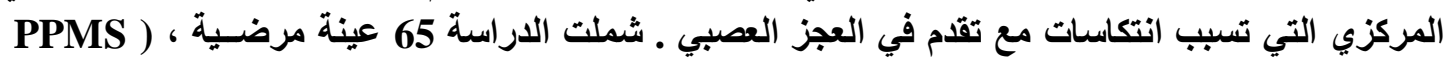
(7,SPMS 21, RRMS 37 افراد اصحاء ظاهرياً ـ نتائج البحث اظهرت وجن من هوض مستويات قليلة من الاجسام المضادة الذاتية للمايلين

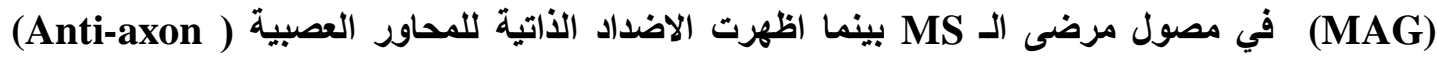

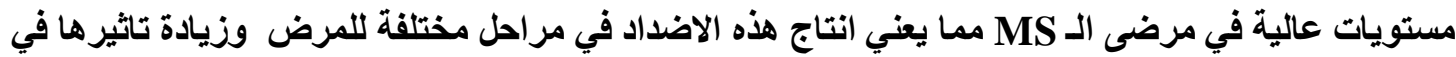

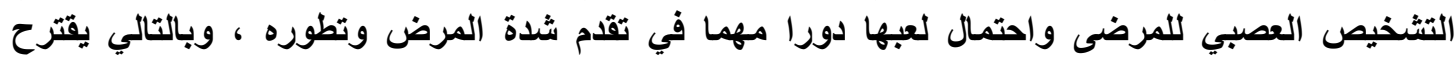

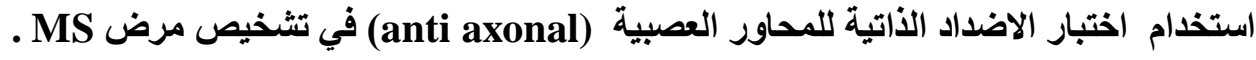

\section{Introduction}

1.1: Multiple sclerosis (MS) is a disease in which the nerves of the central nervous system (brain and spinal cord) degenerate. It is an inflammatory demyelinating disorder of the central nervous system (CNS) and the most common cause of neurologic disability in young adults[1]. MS is autoimmune disease in which an immunologic response to myelin proteins of the CNS is triggered by one or more exogenous agents in a genetically susceptible individual [2]. The disease is characterized by regions of demyelination of varying size and age scattered

Key words: Multiple Sclerosis, anti-Myelin auto-antibodies, anti-axonal auto-antibodies الكلمات المفتاحية: مرض تصلب الاعصاب المتعدد،الاجسام المضادة الأتية للمايلين ،الاجسام المضادة الذاتية للمحاور العصبية. 
throughout the white matter of the CNS, especially in the cerebrum, brainstem, optic nerves, and spinal cord [3]. Clinically, it is characterized by recurrent or chronic progressive neurologic dysfunction[4]. Typically, it presents in young adults between 20 and 40 years with a peak at 30 and the female to male ratio is 2:1[5].

\subsection{Disease Pattern}

There are different clinical manifestations of MS. During an attack, a person experienced a sudden deterioration in normal physical abilities that may range from mild to severe. This attack, sometimes referred to as an exacerbation of MS, typically lasts more than 24 hours and generally more than a few weeks (rarely more than four weeks) [4].

About (65-80)\% of individuals begin with relapsing-remitting (RR) MS, which is the most common type. In this type, they experience a series of attacks followed by complete or partial disappearance of the symptoms (remission) until another attack occurs (relapse), It may be weeks to decades between relapses [6].

In primary-progressive (PP) MS, there is a continuous, gradual decline in a person's physical abilities from the outset rather than relapses. About (10-20)\% of individuals begin with PP-MS[6].

Those beginning with RR-MS can then enter a phase where relapses are rare but more disability accumulates, and are said to have secondary-progressive (SP) MS. About $50 \%$ of RR-MS individuals will develop SP-MS within 10 years. Over several decades, most RR-MS persons will experience progression to SP-MS. ProgressiveRelapsing (PR) MS is a type of MS characterized by a steady decline in abilities accompanied by sporadic attacks [7]. There are cases of MS that are mild and can be recognized only retrospectively after many years and also rare cases of extremely rapid progression of MS symptoms (sometimes fatal) known as malignant or fulminant (Marburg variant) MS [7].

\section{3: Autoantibodies in MS}

\subsection{1: Anti-myelin associated glycoprotein (anti-MAG):}

MAG is one of the main potential myelin sheath auto-antigens; extracellular exposure makes it accessible to antibodies (anti-MAG) [8] Elevated anti-MAG antibodies may predict progression and may also correlate with disease activity (e.g. suggests active demyelination) [8]. Low concentrations of anti-MAG autoantibodies can also be found in patients with MS, SLE and healthy individuals [9].

\subsection{2:Anti-axon antibodies}

Antibodies to axonal cytoskeletal proteins (neurofilaments-NFL) may be markers of axonal damage, as well as important contributors to neurodegeneration and clinical disability in MS [10]. Elevated levels of NFL-specific antibodies are present in sera of patients with PP-MS, and these antibodies are also increased in sera of patients with other neurologic disease [11].

\subsection{Aim of the study}

This study was done to shed some light on:

1. Immunologic detection of anti-myelin (anti-MAG) and anti-axonal antibodies in sera of all study groups. 
2. Investigate whether progression in MS is associated with antibodies to axonal and compare this with antibodies to MAG.

\section{Materials and methods}

\section{1: Subjects}

\subsection{1: Patients}

This prospective study included 65 MS patients (37 RRMS, 21 SPMS, 7PPMS). The genders of the patients were 24 males and 41 females and their age range was from 19 to 60 years. Those patients were recruited from the multiple sclerosis units in Baghdad Teaching Hospital, during the period from April to the end of June 2008. The diagnosis in each case was established by clinical specialists in neurology based clinical radiologic finding, and laboratory investigations.

\subsection{2: Controls}

Fifty apparently healthy individuals (17 males and 33 females) were enrolled in this study, and their age ranged from (18-60) years. Those individuals were recruited from donors of the Central Blood Bank in the Medical City Complex, Baghdad.

\section{2: Materials}

2.2.1: Immunofluorescence assay kit for detection of IgG auto-antibodies against myelin sheaths and myelin associated glycoprotein (anti-MAG) in serum. (BINDING SITE COM-UK).

\subsubsection{1: Kit contains}

Slides, Conjugate, Positive control, Negative control, Salt for PBS pH 7.2 , 1\% Evans blue, Mounting medium, Cover glasses, Blotters.

\section{3: Methods}

\subsection{1: Blood sample collection and preparation:}

Five militaries $5 \mathrm{ml}$ venous blood were obtained from each subject included in the study and placed in a sterile plain tube, then centrifuged and the serum was separated and stored at $-20^{\circ} \mathrm{C}$.

\subsection{2: Immunofluorescence test for detection of IgG auto-antibodies against} myelin sheaths (anti-MAG) and against nerve axon in serum.

The test was performed in immunology department of teaching laboratories, Baghdad medical city, following the procedure protocol included within the kit packing as issued from the manufacturer company.

\subsubsection{1: Test principle:}

The slides were used as an indirect Immunofluorescence technique [12]. The patient samples and appropriate controls were incubated with the sections. The unreached antibodies were washed off and then appropriate Fluorescein-labelled conjugates were applied[12]. Unbound conjugate was washed off as before. Slides were viewed with a fluorescence microscope and positive samples produce apple-green fluorescence which corresponds to areas of the section where autoantibody has bound [13].

\subsubsection{2: Test procedure}

Preliminary preparations:

- Slides and reagents: All reagents and the slides were allowed to reach the room temperature $(20-25)^{\circ} \mathrm{C}$. 
- PBS: was proved as an x20 concentrate, so it was diluted to $1 / 19$. The PBS was used as a diluents for patient sera and also as a washing buffer.

Step 1: Dilution of patient samples with PBS.

Patient samples were diluted to $1 / 10$ by adding $20 \mu \mathrm{l}$ of serum to $180 \mu \mathrm{l}$ of PBS buffer.

Step 2: Addition of diluted samples to substrate slides.

After substrate slides were reached room temperature, we removed it from pouches, labeled it appropriately and placed it in the humid chamber. Then, we added a drop of positive and negative controls to appropriate wells and $50 \mu \mathrm{l}$ of diluted patient samples to the remaining wells.

Step3: Slides incubation.

The slides were incubated for 30 minutes in humid chamber at room temperature.

Step 4: Slides washing with PBS.

The slides were removed from humid chamber and rinsed briefly with PBS by using squeeze bottles. Then, the slides were placed in a rack and immersed in PBS for 5-10 minutes.

Step 5: Addition of fluorescent conjugate to substrate slides.

We were shake off excess PBS and blot around wells. Then, the slides were returned to humid chamber and immediately we covered each well with a drop of anti-Human IgG FITC conjugate.

Step6: Slides incubation in dark chamber.

The slides were incubated for 30 minutes in humid chamber at room temperature, in the dark.

Step 7: Slides washing with PBS and counter stain.

The slides were removed from humid chamber and rinsed briefly with PBS. Then, 2-3 drops of 1\% Evans blue counter stain were added for each $100 \mathrm{ml}$ of PBS prior to slide immersion. After that, the slides were placed in a rack and immersed in blue PBS for 5-10 minutes.

Step 8: Mounting with cover slips.

One slide was removed at a time from PBS wash. Then, quickly dried around the wells and added a drop of mounting medium to each well. Carefully the slide was covered with the cover slips, avoiding air bubbles but, if present, did not attempt to remove. The excess medium from around edge of cover slips was wiped.

Step 9: View slides under fluorescence microscope.

Finished slides were viewed as soon as possible but may be stored for at 2-8 $\dot{\mathrm{C}}$ in dark for up to 3 days, without significant loss of fluorescence [14].

\subsubsection{3: Kit Controls (Results)}

\section{Quality control:}

The positive control was given bright apple-green fluorescent staining of the myelin sheaths, appearing as halos of fluorescence around each axon [14]. The negative control was showed dull green staining in all the tissue, with no discernible fluorescence. While anti-axonal antibodies was given a bright apple-green fluorescent 
staining in the centre of the nerve. If controls did not appear as described, the test was regarded invalid and repeated [15].

\section{Negative}

A sample was considered negative if specific myelin sheath or axon staining was equivalent to or less than the negative control well.

\section{Positive}

A sample was considered positive if myelin sheath or axon staining was observed to be greater than the negative control well, and a clear discernable pattern can be seen in most of the sheaths [15].

\section{4: Statistical Analysis}

Statistical Package for Social Sciences (SPSS) version 15 was used for data entry and analysis. Results were expressed in simple statistical terms. Finding $\mathrm{P}$ value less than 0.05 was considered significant.

Results

\section{1: Sociodemographic characteristics of the study groups:}

The prospective study included 65 (56.5\%) patients with different patterns of MS, RRMS 37(32.1\%), SPMS 21(18.3\%) and PPMS 7(6.1\%). Also 50 (43.5\%) healthy individual served as control, Table (3.1).

Table (3.1): Distribution of study groups

\begin{tabular}{ccc}
\hline Study group & No. & Percentage \\
RRMS & 37 & 32.1 \\
SPMS & 21 & 18.3 \\
PPMS & 7 & 6.1 \\
Control & 50 & 43.5 \\
Total & 115 & 100 \\
\hline
\end{tabular}

Relapsing-remitting: RRMS

Secondary-progressive: SPMS

Primary-progressive: PPMS

\section{2: Sero-immunological data}

3.2.1: Immunofluorescence test for detection of IgG anti-myelin and anti-axon auto-antibodies in all study groups.

Table (3.2) and figure (3.1), demonstrate the results of IFA test for IgG anti-myelin sheath auto-antibodies of all study groups, in which the sero-positive results were 3(14.3\%) in SPMS and 1(14.3\%) in PPMS while in RRMS and controls were zero.

Table (3.3) and figure (3.2), demonstrate the results of IFA test for IgG anti-axon auto-antibodies of all study groups, in which the sero-positive results were 19(90.5\%) in SPMS , 15(40.5\%) in RRMS and 5(71.4\%) in PPMS while in control were 6(12\%). 
Table (3.2): Distribution of IgG anti-myelin auto-antibodies in MS patients and controls.

\begin{tabular}{|c|c|c|c|c|c|c|}
\hline \multirow{4}{*}{$\begin{array}{l}\text { Study } \\
\text { group }\end{array}$} & \multicolumn{4}{|c|}{ Anti-myelin sheath Abs } & \multirow{2}{*}{\multicolumn{2}{|c|}{ Total }} \\
\hline & \multicolumn{4}{|c|}{$($ anti-MAG)* } & & \\
\hline & \multicolumn{2}{|c|}{ Positive } & \multicolumn{2}{|c|}{ Negative } & \multirow[b]{2}{*}{ No. } & \multirow[b]{2}{*}{$\%$} \\
\hline & No. & $\%$ & No. & $\%$ & & \\
\hline RRMS & 0 & 0 & 37 & 100 & 37 & 100 \\
\hline SPMS & 3 & 14.3 & 18 & 85.7 & 21 & 100 \\
\hline PPMS & 1 & 14.3 & 6 & 85.7 & 7 & 100 \\
\hline Control & 0 & 0 & 50 & 100 & 50 & 100 \\
\hline Total & 4 & 3.5 & 111 & 96.5 & 115 & 100 \\
\hline
\end{tabular}

* $P$ value is 0.3718, considered not significant.

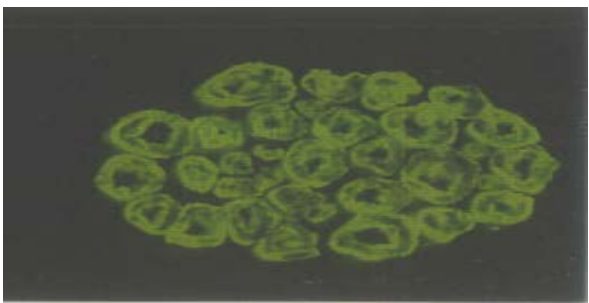

Fig (3. 1): anti-MAG antibodies on monkey peripheral nerve at high power showing the characteristic Immunofluorescent staining of the myelin sheaths (cylindrical shape).

Note: Apple green fluorescent color reflects positive results while those who fail to have the stain considered negative.

Table (3.3): Distribution of IgG anti-axon auto-antibodies in MS and controls.

\begin{tabular}{ccccccc}
\hline & \multicolumn{3}{c}{ Anti-axon Abs } & \multicolumn{2}{c}{ Total } \\
Study group & \multicolumn{2}{c}{ Positive } & \multicolumn{2}{c}{ Negative } & NO. & $\%$ \\
& NO. & $\%$ & NO. & $\%$ & NO & (15) \\
RRMS & 15 & 40.5 & 22 & 59.5 & 37 & 100 \\
SPMS & 19 & 90.5 & 2 & 9.5 & 21 & 100 \\
PPMS & 5 & 71.4 & 2 & 28.6 & 7 & 100 \\
Control & 6 & 12 & 44 & 88 & 50 & 100 \\
Total & 45 & 39.1 & 70 & 60.9 & 115 & 100 \\
\hline
\end{tabular}

${ }^{* *} \mathbf{P}$ value is 0.00021 , considered very significant.

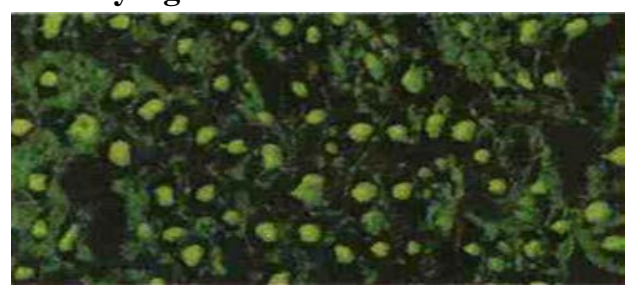

Fig (3. 2): Non-specific anti-axon antibodies on monkey peripheral nerve at high power showing the characteristic Immunofluorescent staining of the neuronal axons (central staining) without myelin sheath staining.

Note:Apple green fluorescent color reflects positive results while those who fail to have the stain considered negative.

\section{Discussion}

In this study, we used an indirect Immunofluorescence (IIF) method to determine the prevalence of anti-myelin, anti-MAG, and anti-axonal auto antibodies in patients with MS and healthy controls,14,3\% Of PPMS and 14,3\% 0f SPMS were sero-positive for anti-MAG antibodies while all RRMS patients and HC were sero-negative. [16]found nearly similar results by using enzyme linked immune-sorbent assay (ELISA) while in 
Moller (1986) [17]study, a higher results were obtained; 20.4\% of PPMS, 17.2\% of SRMS and $3.1 \%$ of RRMS were sero-positive for anti-MAG antibodies by Radioimmunoassay (RIA) which is a very sensitive method. The results suggested that there is a low level of humeral immunity to MAG in MS patients that can only be detected by the most sensitive methods (as in Mollar study) [17]. This weak immune response to MAG may be secondary to the demyelinating process, but could play a role in the progression of the disease.

In screening for anti-axonal antibodies (non-specific), the sero-positivity was $90,5 \%$ in SPMS, $71,4 \%$ in PPMS, $40,5 \%$ in RRMS and $12 \%$ in HC. MS patients had a significantly higher antibody response than HC (sero-positivity was $57.4 \%$ in MS and $12 \%$ in $\mathrm{HC}$ ), and within MS population, we found significantly higher responses in SPMS(90.5\%) in comparison to PPMS 71.4\% and a tendency for higher antibody responses when compared to patients with RRMS 40.5\%. These results agree with Silber (2002) [18] but disagree with [19] which found higher antibody response in PPMS (93.6\%) than SPMS (77.9\%). This difference may be due to use of ELISA which is a more sensitive method.

Using IIFA, this study found elevated serum antibodies to axonal antigens (anti-axon) in subjects with progressive MS [20]. It is feasible that increased amount of axonal proteins are released with axonal degeneration and that may behave as antigens. The elevated level of anti-axon antibodies may be a consequence of the ongoing axonal damage. Potentially increasing the level of antigens present and thereby contributing to the immune response to these axonal proteins. Elevated antibodies in progressive MS suggest a rise in antibody related to ongoing axonal destruction. The possibility that anti-axon antibodies may play a more direct pathogenic role in progressive MS should be considered [20].

\section{Conclusion:}

1. Low level of auto-antibodies to MAG in Iraqi patients with MS.

2. Anti-axon were elevated in progressive MS suggest that production of these antibodies may reflect ongoing neurodegenerative process.

3. The differences between the response to MAG and anti-axon auto-antibodies suggest some degree of selectivity in the humeral immune response.

4. Our findings support the use of serum anti-axon as a marker for axonal destruction.

\section{References}

1. Baliga, R. (2007). Central nervous system. In: Crash Course: Internal Medicine, Elsevier Mosby. 34:309-332

2. Blumhardt, L X., conttantnescu, CS. (2004). Multiple sclerosis. In: Clinical neurology, United Kingdom. 20:400-402.

3. Turgeon, ML. (2003). Autoimmune Disorders. In: Immunology \& Serology in Labrotory Medicine. 3rd ed, mosby. 25:373-399.

4. Lindsey, JW., Wolinsky, JS. (2007). Demyelinating Diseases. In: ACP MEDICINE. $3^{\text {rd }}$ ed. 11:633-712.

5. Dangond, F. (2000). Multiple sclerosis. In: Basic and clinical immunology. $2^{\text {nd }}$ ed, $17: 244-248$. 
6. Langer-Gould, A., Popat, RA., Huang, SM., et al. (2006). Clinical and Demographic Predictors of Long-term Disability in Patients with Relapsing-Remitting Multiple Sclerosis: A Systematic Review. Arch Neurol. 63:1686.

7. Confavreux, C., Vukusic, S., Moreau, T., Adeleine, P. (2000). Relapses and progression of disability in multiple sclerosis. N Engl J Med. 343:1430.

8. Berger, T., Rubner, P., Schautzer, F. et al. (2003). Antimyelin antibodies as a predictor of clinically definite multiple sclerosis after a first demyelinating event. $\mathrm{N}$ Engl J Med. 349:139.

9. Amor, S., Giovannoni G. (2007). Antibodies to myelin oligodendrocyte glycoprotein as a biomarker in multiple sclerosis — are we there yet? Mult Scler. 13:1083-1085.

10. Dutta, R., Trapp, BD. (2007). Pathogenesis of axonal and neuronal damage in multiple sclerosis. Neurology. 68:22-31.

11. Griffith, I. (2008). Axonal swelling and degeneration in multiple sclerosis. Science. 280: 1610-13.

12. Mattews, WB., Acheson, ED., Bachelor, JR. et al. (1985). Multiple sclerosis. In: McAlpine's textbook of neurology, $2^{\text {nd }}$ ed, Churchill Livingstone. 5: 65-74.

13. Lisak, RP., Zwiman, B., Norman, M. (1975). Antibodies in Neurologic Diseases: Immunofluorescent Demonstration. Arch Neurol, March 1, 32(3): 163 - 167.

14. Ayad, IM. (2001). Autonomic dysfunction in patients with multiple sclerosis. Athesis submitted to the Iraqi council for medical specializations for the F.I.C.M.S in Neuromedicine.

15. Heinzlef, O., Alamowitch, S., Sazdovitch, V., et al. (2006). Autoimmune diseases in families of French patients with multiple sclerosis. Acta Neurol Scand. 101:36.

16. Nobile-Orazio, Eduardo, JF., McIntosh, G., et al. (1985). Anti-MAG antibody and antibody complexes: Detection by radioimmunoassay. Neurology. 35(7):988-992

17. Moller, JR., (1989). Antibodies to myelin-associated glycoprotein (MAG) in the cerebrospinal fluid of multiple sclerosis patients. J Neuroimmunol. Mar; 22(1):55-61.

18. Silber, E. (2002). Patients with progressive MS have elevated antibodies to NF subunit. Neurology. 58:1372-81.

19. Ehling, R. (2004). Increased frequency of serum antibodies to NF-L in patients with primary progressive MS. Multiple sclerosis. 10: 601-6.

20. Rawes, JA., (2002). Antibodies to neuronal antigens in sera of patients with MS. Mult sclera. 3: 363-9. 